\title{
两亲性体系自组装机制的多尺度模拟及动力学 网络模型分析
}

\author{
郑小燕 ${ }^{1 \dagger *}$, 曾祥泽 ${ }^{2 \dagger}$, 李占伟 ${ }^{3}$
}

1. 北京理工大学化学与化工学院, 光电转换材料北京市重点实验室, 原子分子簇科学教育部重点实验室, 北京 100081

2. 圣路易斯华盛顿大学生物医学工程系, 圣路易斯, 密苏里州, 63130, 美国

3. 中国科学院长春应用化学研究所, 高分子物理与化学国家重点实验室, 长春130022

†同等贡献

*通讯作者, E-mail: xiaoyanzheng@bit.edu.cn

收稿日期: 2020-05-15; 接受日期: 2020-06-17; 网络版发表日期: 2020-08-13

摘要两亲性构筑基元自组装在制备纳米功能材料中有着不可替代的重要性. 在微观和介观尺度上对自组装 过程内在机制的认识对实现纳米自组装结构的精准调控起着关键作用. 多尺度模拟可实现在微观和介观尺度上 对构筑基元自组装过程的动力学模拟, 获取亚稳态、稳态的组装构型及动力学轨迹. 基于所得动力学轨迹, 可进 一步构建动力学网络模型, 获得自组装过程的动力学转移路径, 为设计新的自组装构筑基元和调控自组装结构提 供理论依据. 本文主要综述了我们近期通过多尺度模拟结合动力学网络模型分析来研究两亲性构筑基元(如有机 小分子、嵌段共聚物和补丁粒子)自组装内在机理方面的研究进展.

关键词两亲性分子, 自组装, 多尺度模拟, 动力学网络模型

\section{1 引言}

两亲性构筑基元由亲水和疏水两部分组成，如表 面活性剂、星型嵌段共聚物和补丁粒子等, 它们可在 水溶液中自组装形成多种结构，如胶束、囊泡、纳米 管、笼状结构等. 这些自组装结构是在各种弱的非共 价键相互作用力 (氢键、 $\pi-\pi$ 、静电和疏水效应等)协同 下由热力学和动力学共同驱动形成的 ${ }^{[1 \sim 4]}$. 分子间弱相 互作用的协同效应决定分子自组装结构的热力学稳定 性. 通过调节外界的光、电、磁、温度、浓度、溶剂
环境等条件可调控两亲性构筑基元的两亲性, 并改变 分子间各种弱相互作用, 调节自组装过程的动力学路 径, 实现可控自组装和解组装 ${ }^{[1 \sim 4]}$. 自组装过程中热力 学和动力学都非常重要, 可控自组装的实现, 既要考虑 体系的热力学性质, 也要考虑其动力学信息 ${ }^{[5,6]}$.

多尺度动力学模拟(包括全原子分子动力学、粗 粒化分子动力学、布朗动力学、耗散粒子动力学等) 可以实现在微观层次上对两亲性构筑基元间弱相互作 用的描述，并得到在介观层次上纳米聚集体形成的动 态过程, 同时获取两亲性体系自组装过程中热力学上

引用格式: Zheng X, Zeng X, Li Z. Multiscale simulation and kinetic network model analysis of the self-assembly of amphiphilic systems. Sci Sin Chim, 2020, 50: 1118-1131, doi: 10.1360/SSC-2020-0082 
各种亚稳态结构及平衡构型，是研究分子自组装内在 机制的有力工具 ${ }^{[7 ~ 9]}$. 动力学模拟通过数值求解牛顿运 动方程来获取体系的热力学性质及动力学信息 ${ }^{[10]}$. 所 研究体系可以用不同分辨率的模型表示. 全原子模型 中，每个原子用一个粒子来表示，因此具有原子级别 的分辨率. 但由于计算资源的限制，所能研究的空间 及时间尺度有限. 粗粒化模型中, 将多个原子或基团 分组并用一个粒子代替，减少整体自由度，从而模拟 可以达到更大的空间及时间尺度. 然而，分子自组装 过程层次复杂，具有多尺度的特征：自组装过程既涉 及构筑基元之间的分子间弱相互作用，也涉及组装体 之间在大尺度上融合、生长等过程，表现出不同的特

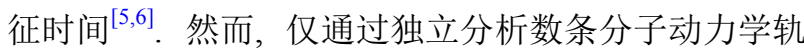
迹很难得到具有统计意义的动力学信息, 如不同亚稳 态之间的自由能势垒及跃迁概率、分子组装体形成过 程主要动力学路径及各路径所占比例.

蛋白质折叠在生命机制中起着十分重要的作用. 对于可折叠蛋白来说, 主要存在两个态, 即无定型的非 折叠态(unfolded state)和天然构象态(native state). 对于 非折叠态，蛋白质构象相对无序，可采取构象比较多， 因此熵较大. 同时, 由于未形成特定的二级结构, 势能 较高; 而当蛋白质处于天然构象态时, 蛋白序列中残基 之间的氢键相互作用以及与溶剂之间的亲疏水相互作 用等, 使其形成特定的二级甚至三级结构, 体系的势能 大大降低, 与此同时, 体系的构象数量也明显减少, 熵 也较小. 因此, 科学家们通常采用漏斗状势能面 (funnel-shaped energy landscape)来描述蛋白质折叠过 程 ${ }^{[1113]}$. 在此势能面上, 蛋白质天然构象处于势能面最 低点, 与非折叠构象有较大的能量差别. 然而, 非折叠 态有较大的构象熵(configuration entropy), 最终导致天 然构象与非折叠构象有较小的自由能差. 因此, 蛋白质 的折叠和解折叠过程是个可逆的过程. 例如, 2011年 Shaw等 ${ }^{[14]}$ 采用全原子分子动力学模拟一个小的螺旋 状蛋白(Villin headpiece)的折叠和解折叠过程. 通过采 用四种不同的分子力场(FF03、FF99SB、CHARMM27 和CHARMM22)模拟此蛋白折叠过程，在模拟过程中 观察到非折叠构象和天然构象之间的转换高达 50 次, 很显然, 此蛋白的折叠过程具有可逆性. 所得此蛋白的 折叠自由能都非常小，介于 $0.21 ~ 1.0 \mathrm{kcal} / \mathrm{mol}$ ，与实验 值 $(0.8 \mathrm{kcal} / \mathrm{mol})$ 非常接近，而其对应过程的焓变分别 为: $9.7 、 19.7 、 19.3$ 和 $17.0 \mathrm{kcal} / \mathrm{mol}$, 后三种力场参数
所得焓变与实验值 $(25 \mathrm{kcal} / \mathrm{mol})$ 更接近一些. 因此, 蛋 白折叠前后焓变很明显, 而自由能差很小. 然而, 自组 装体系中可能存在多种不同能量的亚稳态，不同亚稳 态之间有较大的自由能势垒, 因此漏斗状势能面并不 适用于描述分子自组装过程. 同时，自组装结构与初 始分散的构筑基元之间的自由能差别要远远大于蛋白 质天然构象与非折叠构象之间的差别, 从而导致自组 装过程不可逆. 例如, 2015年Frenkel等 ${ }^{[15]}$ 通过理论预 测表明, 在318.5 K时, 86块DNA砖块模型(DNA brick) 自由单体到组装形成三维纳米结构过程中的自由能差 大约为 $70 k_{\mathrm{B}} T$ ( $k_{\mathrm{B}}$ 代表玻尔兹曼常数), 远大于之前同等 大小的蛋白质折叠自由能.

近年来, 动力学网络模型(kinetic network model, KNM)中的马尔可夫态模型(Markov state model, MSM $)^{[16-21]}$ 已被广泛用于研究处于平衡态体系的动力 学过程, 如蛋白质折叠 ${ }^{[22 ~ 24]} 、$ DNA 到RNA的转 录 $^{[25 ~ 28]}$ 、蛋白质受体与小分子配体的结合 ${ }^{[29 \sim 33]}$ 、寻 找蛋白质-小分子结合位点 ${ }^{[34]}$ 及多肽聚合 ${ }^{[35 \sim 38]}$. 在此模 型中, 体系的动力学过程被近似成离散状态、离散时 间的马尔可夫过程. 体系的构象空间被划分成不同小 态(microstate), 相比于不同小态, 同一小态中的构象具 有更高的相似性. 基于小态间的转移概率矩阵(transition probability matrix, TPM), 可以得到体系的动力学 信息，如态与态之间的平均首次通过时间(mean first passage time). 同时, 还可用转移路径理论(transition path theory, TPT) ${ }^{[39,40]}$ 找出任意两态间的动力学转移 路径(transition path, 以下简称转移路径). 一般情况下, 体系的构象空间会被划分为成百甚至上千个小态，如 此多的小态并不利于直观理解体系的动力学过程和隐 藏其中的机理. 因此, 根据小态之间的动力学信息, 这 些小态将被进一步聚合成大态(macrostate). 至此, 一个 完整的MSM已经建成. 需要注意的是, 只有满足马尔 可夫性的模型才被视为有效模型. MSM一大优势在于 可通过多条时间尺度较短的动力学轨迹来获取体系长 时间尺度上的动力学信息, 从而填补了直接进行分子 动力学模拟所能达到的时间尺度与研究大体系长时间 尺度之间的鸿沟 ${ }^{[17,18]}$.

然而，基于平衡态而发展的构建MSM的方法并不 能直接用于自组装体系 ${ }^{[41]}$. 其主要包括三个问题: (1) 现有划分构象空间的方法，如基于不同构象间原子均 方根距离(root mean square distance, RMSD)的聚类方 
法 $^{[42]}$, 均要求体系中的原子可以相互分辨 ${ }^{[43]}$. 构象 $\mathrm{A}$ 和

$\mathrm{B}$ 之间的RMSD可以表示为 $d(\mathrm{~A}, \mathrm{~B})=\sqrt{\frac{\sum_{1}^{N}\left(r_{i, \mathrm{~A}}-r_{i, \mathrm{~B}}\right)^{2}}{N}}$,

其中, $N$ 为体系的原子数目, $r_{i, \mathrm{~A}}$ 和 $r_{i, \mathrm{~B}}$ 分别为体系中第 $i$ 个原子在构象 $\mathrm{A}$ 和 $\mathrm{B}$ 中的坐标. 然而, 自组装体系中的 单体不可分辨, 相互交换后不影响体系的物理化学性 质 ${ }^{[43]}$. 因此, 我们不能直接判定单体的序号, 利用穷举 法, 会得到 $N$ ! 个不同的RMSD. 对于有大量单体的自组 装体系, 复杂度如此高的计算是不切实际的. (2) 由于 自组装体系初始态远离自由能最低点, 大部分自组装 过程是不可逆的. 这种情况下, 有限时间内计算模 拟所得到的体系动力学并不满足细致平衡(detailed balance). 因此, 我们不能直接用TPT方法来寻找不同 态之间转移路径, 限制了我们对体系动力学的深入理 解. (3) 自组装体系可能存在大量并行、概率相似的 转移路径. 由于缺少主导自组装路径(dominant selfassembly pathway), 进一步加大了态转变机理的理解 难度.

近年来, 我们和其他研究者分别提出了相关方法 来解决上述问题, 并发展了针对自组装体系的KNM. 为了解决自组装体系中单体不可分辨的问题, 我们选 取可以反映聚集体特征的参数, 如聚集体大小和非球 形参数(用来刻画聚集体的形貌)来划分构象空间 ${ }^{[44,45]}$. Hagan等 ${ }^{[46]}$ 和Frenkel等 ${ }^{[47]}$ 提出, 用无向图来表示聚 集体, 根据图的拓扑结构来划分构象空间. 为了找出 自组装体系中态与态之间的转移路径, 我们提出了 “质 量流(mass flow)”的概念, 用来表示聚集体在不同小态 间的转移质量 ${ }^{[4]}$. 基于“质量流”矩阵, 我们可以用修 正的Dijkstra算法 ${ }^{[48]}$ 来寻找任意两态间的转移路径. 为 了解决体系中存在大量概率相似转移路径的问题, Meng等 ${ }^{[49]}$ 提出了“路径整合(path lumping)”方法. 此方 法根据转移路径之间的相似性, 把转换路径聚合成少 量 “路径通道”, 从而更直观地理解态与态之间的转变 过程.

本文主要介绍近年来我们通过结合多尺度模拟与 动力学网络模型, 对两亲性构筑基元(如有机小分子、 嵌段共聚物及补丁粒子)自组装过程的热力学组装构 型和动力学路径分析方面的进展, 为实验上新的两亲 性构筑基元设计、自组装过程的调节和自组装结构可 控提供理论依据.

\section{2 两亲性有机小分子自组装机制研究}

\section{1 全原子分子动力学模拟两亲性有机小分子自 组装}

实验发现，两亲性有机小分子AzoC10（1-[10-(4azophenoxy)decyl]pyridinium bromide)分子内部的偶 氮苯基团在紫外可见光照下会发生顺反构型的翻转: 紫外光照射时, 偶氮苯从反式构象转化为顺式构象; 被 可见光照射时, 则从顺式构象转化为反式构象. AzoC10具有两亲性, 可在水溶液中自组装形成囊泡. 加入 $\alpha$-环糊精 $(\alpha-C D)$ 后，反式偶氮苯和 $\alpha-C D$ 空腔尺寸 匹配可形成包合型复合物, 由于 $\alpha-C D$ 外侧亲水而失去 两亲性, 囊泡状聚集体解组装; 在紫外线照射下, 光异 构化转为顺式构型后体积变大, 不能被 $\alpha-C D$ 的空腔包 合, 形成两亲性的主客体复合物, 自组装形成更大的囊 泡 $^{[50]}$.

为了揭示水溶液中两亲性体系光控可逆自组装和 解组装的机制, 我们采用全原子分子动力学的方法来 模拟 $\mathrm{AzoC} 10$ 和 $\alpha-\mathrm{CD}$ 主客体分子间的相互作用. 对主 体 $\alpha-C D$ 和客体AzoC10, 我们分别采用GLYCAM06力 场 $^{[51]}$ 和通用 $A M B E R$ 力场 ${ }^{[52]}$. 为了进一步准确描述 AzoC10顺式和反式构型, 我们用B3LYP/6-31G*方法 拟合用以区分偶氮苯基团顺式和反式构型的二面角 参数.

我们首先模拟了 cis-, trans-AzoC10与主体 $\alpha-\mathrm{CD}$ 的 包合过程动力学以及主客体结合模式. 模拟结果表明, 对于 cis-AzoC10, 由于顺式偶氮苯基团的体积比较大, 其很难被包合在 $\alpha-C D$ 的空腔中. 顺式偶氮苯基团从 $\alpha-C D$ 的小口端进入并穿过空腔, 然后暴露在大口端一 侧的水溶液中, 使得 $c i s-\mathrm{AzoC} 10$ 的烷基链在 $\alpha-\mathrm{CD}$ 的空 腔中来回穿梭; 而对于trans-AzoC10, 无论初始构型如 何, $\alpha-\mathrm{CD}$ 与trans-AzoC10都可形成稳定的主客体复合 物，在偶极-偶极和疏水相互作用驱动下，反式偶氮苯 都沿 $\alpha-C D$ 的中轴线包合在其空腔中 ${ }^{[33]}$.

为更进一步理解主客体间相互作用的强度，我们 采用伞形抽样法(umbrella sampling) ${ }^{[54]}$ 计算 $c i s-$, transAzoC10穿越 $\alpha-C D$ 空腔过程中平均力势 (potential of mean force, PMF)的变化(图1). 在每个伞形抽样模拟 中, $\alpha-\mathrm{CD}$ 和偶氮苯基团在沿 $\alpha-\mathrm{CD}$ 中轴线方向的质心间 距都被限制在某个参考值附近, 且客体分子AzoC10的 烷基链可以自由运动. 通过伞形抽样法获得客体分子 


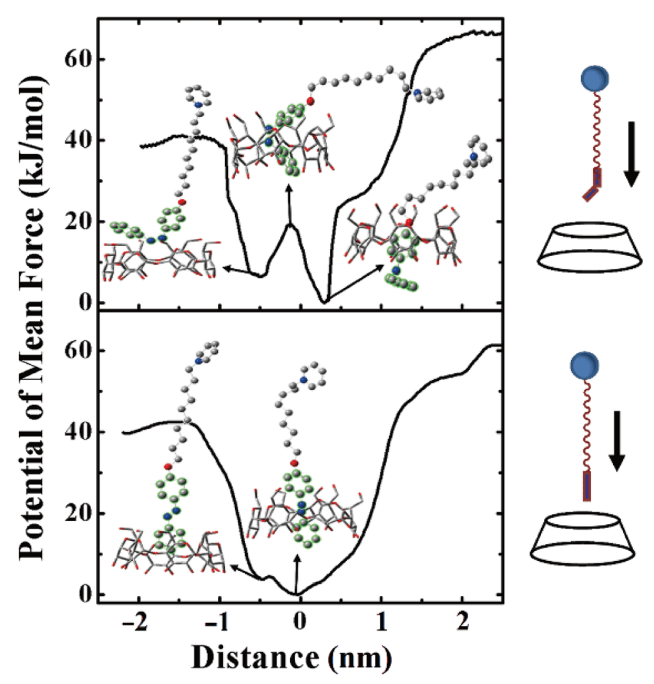

图 1 随着 $\alpha-\mathrm{CD}$ 和偶氮苯基团质心间距的改变, $\mathrm{AzoC} 10$ 穿 越 $\alpha-C D$ 空腔过程的平均力势 (potential of mean force, $\mathrm{PMF})^{[53]}$. 上图: cis-AzoC10; 下图: trans-AzoC10. 沿反应坐 标的一些代表性结构已标注在图中. 右侧示意图中, 红色棒 代表偶氮苯基团，其中弯曲的代表顺式构象，直的代表反式 构象; 蓝色的椭球代表吡啶盐头基; 红色的波浪线条代表 AzoC10的烷烃链(网络版彩图)

Figure 1 Potentials of mean force for AzoC10 to translocate through the cavity of $\alpha-\mathrm{CD}$, as a function of the center-of-mass distance between $\alpha-\mathrm{CD}$ and the azobenzene moiety of AzoC10 [53]. The PMF for cisAzoC10 is shown in the upper panel and that for trans-AzoC10 is shown in the lower panel. In the right cartoon picture, the red bar represents the azobenzene moiety, with the bent one for the cis-isomer and the straight one for the trans-isomer; the blue ellipse represents the pyridinium headgroup; the red string represents the long alkyl chain of AzoC10 (color online).

从 $\alpha-C D$ 一侧穿过空腔到达另一侧过程中PMF的变化. 反应坐标的符号法则定义方法如下：偶氮苯基团位于 $\alpha-\mathrm{CD}$ 主面侧，反应坐标为负; 偶氮苯基团位于 $\alpha-C D$ 次 面侧，则为正；当偶氮苯基团质心与 $\alpha$-CD质心重合时， 则为 0 . 对于trans-AzoC10, PMF的能量最小点出现在 $\alpha-C D$ 和偶氮苯基团质心间距为 $0 \mathrm{~nm}$ 处，此时 transAzoC10的偶氮苯基团被包合在 $\alpha-C D$ 空腔中心，此包 合型配合物形成过程基本上是个能量下降过程．将偶 氮苯基团从 $\alpha-C D$ 小口端拉出疏水性空腔需要消耗大 约 $45 \mathrm{~kJ} / \mathrm{mol}$ 的能量. 对于 $c i s-\mathrm{AzoC} 10, \mathrm{PMF}$ 曲线上存在 两个极小点：其中一个偶氮苯基团刚好位于 $\alpha-C D$ 主面 上方的位置, 另一个偶氮苯基团中一个苯环在 $\alpha-C D$ 次 面侧暴露到水溶液中. 两极小值间存在一个能垒, 此时 体积较大的顺式偶氮苯基团刚好位于 $\alpha-\mathrm{CD}$ 空腔中心, 表明位阻效应使得 $\alpha$-环糊精无法与客体分子中偶氮苯 基团形成包合型配合物. 因此， $\alpha-\mathrm{CD} / c i s-\mathrm{AzoC} 10$ 复合
物具有两亲性, 可自组装形成囊泡, 而不具有两亲性的 $\alpha-\mathrm{CD} /$ trans-AzoC10 则解组装 ${ }^{[33]}$.

\section{2 粗粒化分子动力学模拟两亲性有机小分子自 组装}

基于 2.1 节的全原子分子动力学模拟结果，我们进 一步通过粗粒化分子动力学研究大量主体和客体分子 自组装过程，以揭示光可控自组装过程中聚集体形貌 和尺寸对组装单体分子结构和浓度的依赖关系. 大量 主客体同时存在时，体系的动力学行为在时间和空间 尺度上都超出了全原子分子动力学模拟能到达的尺 度, 需要结合粗粒化分子动力学模拟来实现 ${ }^{[55]}$.

本文，我们选择了根据化合物在极性和非极性相 中的配分自由能来参数化的MARTINI力场, 其中体系 的化学本质主要通过极性 $(\mathrm{P})$ 、半极性半非极性 $(\mathrm{N})$ 、 非极性 $(\mathrm{C})$ 和带电 $(\mathrm{Q})$ 四种珠子类型表示 ${ }^{[56]}$. 环状结构 对应的珠子通过加前缀“S”来区分 ${ }^{[56]}$. 此处, 顺式、反 式客体分子均由14个珠子表示(图2(a)). 其中，偶氮苯 基团由 $\mathrm{SC} 4$ 珠子表示, $\mathrm{N}=\mathrm{N}$ 双键由 $\mathrm{N} 0$ 珠子表示, 碳氢链 由 $\mathrm{C} 2 、 \mathrm{C} 1$ 和 $\mathrm{C} 2$ 三个珠子表示，吡啶盐基团则由 $\mathrm{SC} 3$ 、 $\mathrm{SC} 3$ 和 $\mathrm{SQ} 0$ 三个珠子表示. 溴离子用带负电荷的Qa表 示. $\alpha$-CD采用 18 个粗粒化珠子表示，每个糖单元分配 三个珠子，主面上- $\mathrm{CH}_{2} \mathrm{OH}$ 基团用 $\mathrm{P} 1$ 表示，次面的两 个-CHOH用P4表示(图2(a)). 根据研究体系的特点, 我 们对MARTINI力场参数做了相应修改. 在粗粒化模型 中, 为了区分偶氮苯的顺式和反式构型, 我们采用简谐 势能函数 $V_{\text {angle }}(\theta)=1 / 2 \times K_{\text {angle }}\left(\theta-\theta_{0}\right)^{2}$ 来定义由 $\mathrm{SC} 4 、 \mathrm{~N} 0$ 和 $\mathrm{SC} 4$ 三个珠子所形成的角，其中顺式和反式偶氮苯 对应的 $K_{\mathrm{angle}}$ 分别赋值为 10 和 $30 \mathrm{~kJ} /\left(\mathrm{mol} \mathrm{rad}^{2}\right)$; 平衡角 度 $\theta_{0}$ 分别设为 $120^{\circ}$ 和 $180^{\circ}$. 为了保持 $\alpha-\mathrm{CD}$ 空腔的刚性, 我们对 $\alpha-C D$ 的所有键长项均施加适当的限制，且每个 角度都分配相对较大的力常数. 此外, 还适当引入二面 角项. 对于水溶剂, 直接采用标准的MARTINI力场 模型。

对于 cis-AzoC10和trans-AzoC10, 我们分别构建了 不同浓度的模拟体系, 结果发现: 当客体分子浓度较低 $(N=500)$ 时, cis-AzoC10和trans-AzoC10最终都从小聚 集体转化为球形胶束; 而当浓度较高 $(N=2000)$ 时, 水 溶液中不同形貌和尺寸的聚集体共存，其中最大聚集 体的形貌存在明显差异. cis-AzoC10形成类盘状胶束, 而trans-AzoC10则呈蠕虫状胶束. 我们同时对聚集体 

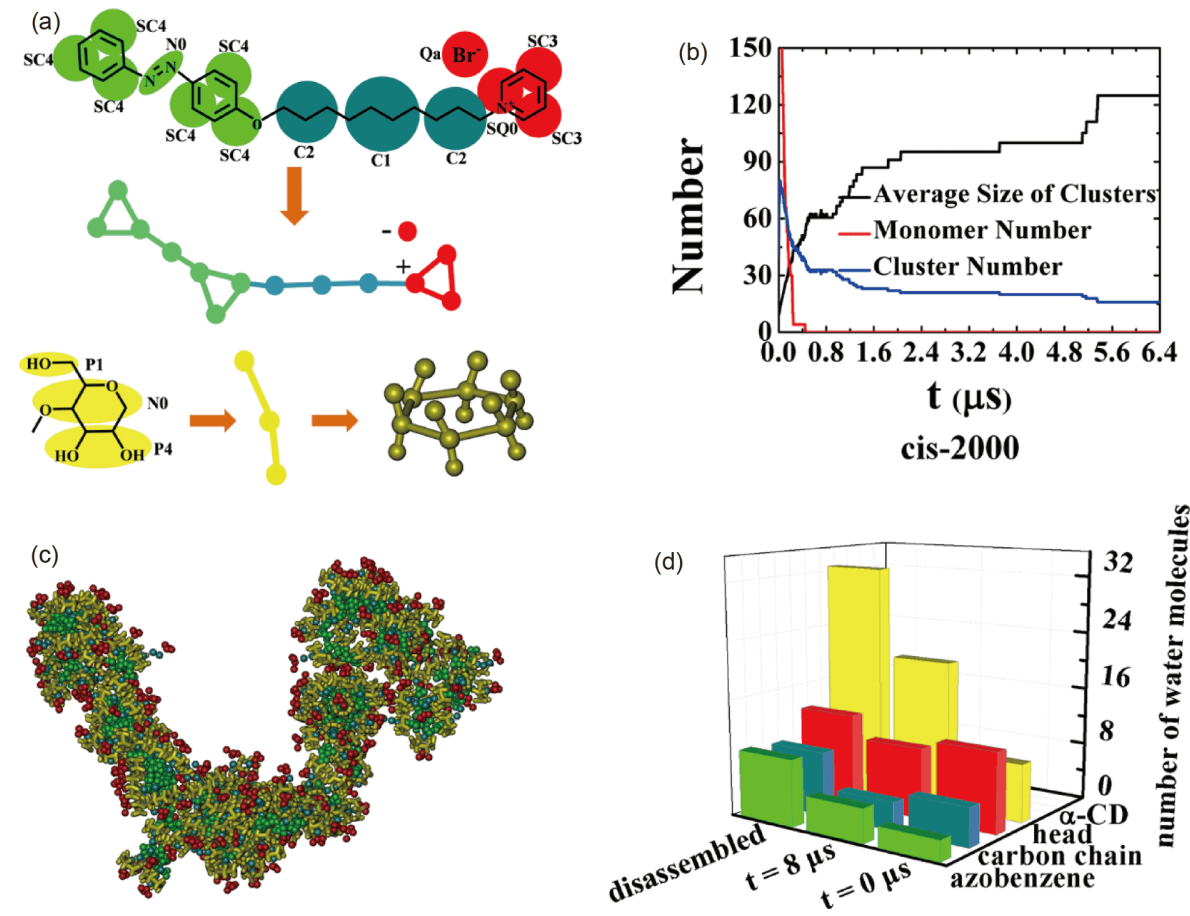

图 2 (a) trans-AzoC10和 $\alpha-\mathrm{CD}$ 从全原子结构到粗粒化珠子的映射关系. 珠子类型已在图中标出，其中前缀“S”代表环状结构 的珠子类型. 最终粗粒化模型以球棍形式表示. (b) 在聚集过程中, 表面活性剂 $c i s-A z o C 10$ 总数 $N=2000$ 时的体系中单体数目、 聚集族数目以及聚集体尺寸随着时间演化的变化曲线. (c) 由 $c i s-A z o C 10 / \alpha-C D$ 形成的类蠕虫状胶束的快照. 为清晰起见, 盒子 中的CG水珠子没有显示. (d) trans-AzoC10/ $\alpha-C D$ 解组装模拟起始 $(t=0 \mu \mathrm{s})$, 最终 $(t=8.0 \mu \mathrm{s}$ )及完全“解组装”状态时(通过在水盒子 中随机分布配合物trans-AzoC10/ $\alpha-\mathrm{CD}$ 后的平衡过程得到)，偶氮苯、碳链、头部基团和 $\alpha-\mathrm{CD}$ 周围 $5 \AA$ 范围内水分子的分布情 况. $(\mathrm{a}, \mathrm{c}, \mathrm{d})$ 中偶氮苯基团、烷基链和亲水头部、 $\alpha-C D$ 分别使用绿色、青色、红色和黄色表示 ${ }^{[55]}$ (网络版彩图)

Figure 2 (a) Mapping from atomistic structures to CG beads for trans-AzoC10 and $\alpha$-CD. The types of beads are labeled with the prefix "S" denoting the ring beads. The final CG models are represented by the ball-and-stick scheme. (b) Time evolution of the number of monomers, the number of clusters, and the average size of clusters in the aggregation of cis-AzoC10 with $N=2000$. (c) The snapshot of worm-like micelle formed by cisAzoC10/ $\alpha$-CD. (d) The atomistic water distributions within $5 \AA$ of each residue: azobenzene, carbon chain, head group and $\alpha-C D$ at the beginning $(t=$ $0 \mu \mathrm{s})$ and end $(t=8.0 \mu \mathrm{s})$ of the simulation, as well as in the completely disassembled state which was obtained by equilibrating randomly distributed trans-AzoC10/ $\alpha-\mathrm{CD}$ in a box of water. In $(\mathrm{a}, \mathrm{c}, \mathrm{d})$, the azobenzene, carbon chain, head group and $\alpha-\mathrm{CD}$ are displayed in green, cyan, red and yellow, respectively [55] (color online).

形成动力学过程进行了分析, 并发现聚集体的形成过 程大致分为三个阶段：第一阶段, $t<0.03 \mu \mathrm{s}$, 球状胶束 成核过程. 此阶段体系中聚集体的数目急剧增加, 在数 十纳秒后达到最大值, 与此同时单体数目急剧减少. 第 二阶段, $0.03 \mu \mathrm{s}<t<0.3 \mu \mathrm{s}$, 球状胶束形成和长大过程. 此阶段分子聚集动力学服从指数规律 $t^{0.5}$, 这与 Smoluchowski扩散限制聚集理论一致(图2(b) $)^{[57]}$. 第三阶段, 由于带电头部基团间存在排斥作用，通过融合来增长 聚集体尺寸的速度大幅变缓. 此阶段对应类盘状胶束 或类蠕虫状胶束形成过程(图2(b) $)^{[55]}$.

接下来, 我们进一步研究了主客体复合物在水溶 液中的自组装和解组装过程. 如图2(c)所示, 通过粗粒 化分子动力学模拟发现, cis-AzoC10/ $\alpha-C D$ 能够在水溶
液中自组装形成蠕虫状胶束，其中疏水性偶氮苯基团 聚集形成蠕虫状胶束的疏水内核, 亲水性吡啶盐位于 蠕虫状胶束最外侧, 大部分 $\alpha-\mathrm{CDs}$ 都穿梭到 $c i s-\mathrm{AzoC} 10$ 的吡啶盐一侧, 使得疏水性偶氮苯基团完全露在 $\alpha-C D$ 空腔外部以保持两亲性. 为模拟trans-AzoC $10 / \alpha-\mathrm{CD}$ 解 组装过程，我们通过PACKMOL预组装了主客体复合 物组成的球形胶束模型 $(N=40)$, 并做了 $8 \mu \mathrm{s}$ 的粗粒化 分子动力学模拟. 与此同时, 我们将同等数量 $(N=40)$ 的主客体复合物自由分散于相同大小的溶剂盒子中进 行了 100 ns对比模拟，并将最终构型标记为“解组装”. 如图2(d)所示, 在模拟过程的起始、最终和完全“解组 装”三种情况下，体系中各组分(偶氮苯、碳链、吡啶 盐和 $\alpha-C D)$ 在 $5 \AA$ 范围内水分子分布情况发生明显变 
化. 可以看出, $t=8 \mu \mathrm{s}$ 时, 体系中各基团周围水分子的分 布情况与“解组装”对照组更接近，说明预组装球形胶 束在模拟过程中会自发解组装 ${ }^{[55]}$, 解释了实验上两亲 性主客体复合物光控可逆组装和解组装的内在机制 ${ }^{[50]}$.

\section{3 构建动力学网络模型分析两亲性有机小分子 自组装路径}

两亲性有机小分子PYR 和PYN在结构上具有高度 的相似性(图3(a,b)), 但PYR最终自组装形成纳米管而 PYN则组装成囊泡, 其组装体形貌差异巨大 ${ }^{[58,59]}$. 单从 两种分子构筑基元结构的细微差别, 很难直接解释纳 米聚集体形貌的差异. 为了揭示引起这种差异的内在 自组装机理, 我们 ${ }^{[45]}$ 通过将粗粒化分子动力学模拟和 动力学网络模型相结合来研究这两种有机小分子初始
阶段的自组装过程. 选取自组装过程中聚集体尺寸以 及非球形参数(刻画聚集体的形貌)来划分态. 基于“质 量流”矩阵，采用修正的Dijkstra算法 ${ }^{\left[{ }^{[8]}\right]}$ 找到了聚集体 自组装过程的动力学路径. 由于得到的动力学路径数 量众多(将近2000条), 我们采用路径整合(path lumping $)^{[49]}$ 方法将这些路径聚合成两条路径通道(path channel): 逐渐增长型通道(incremental channel)和跳跃 型通道(hopping channel). 在逐渐增长型通道中, 聚集 体通过黏附比较小的聚集体或单体逐步增大(图3(c)); 而在跳跃型通道中，聚集体则是和自身大小相似的聚 集体碰撞融合, 呈现出跳跃式的增长(图3(d)).

我们发现，逐渐增长型通道和跳跃型通道同时存 在于PYR 和PYN中, 唯一的区别在于两种通道的比例 不同. 在PYR体系中, 将近 $70 \%$ 的转移路径属于逐渐增
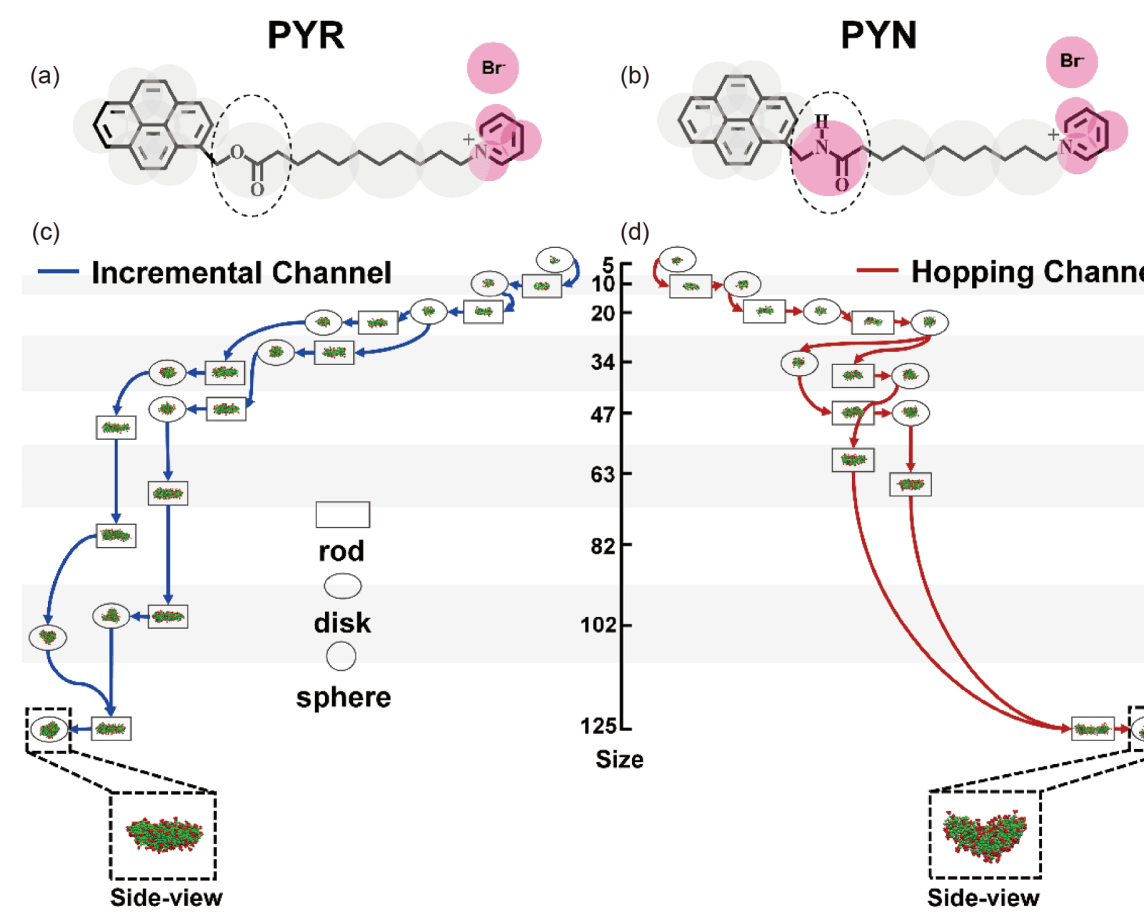

(i)
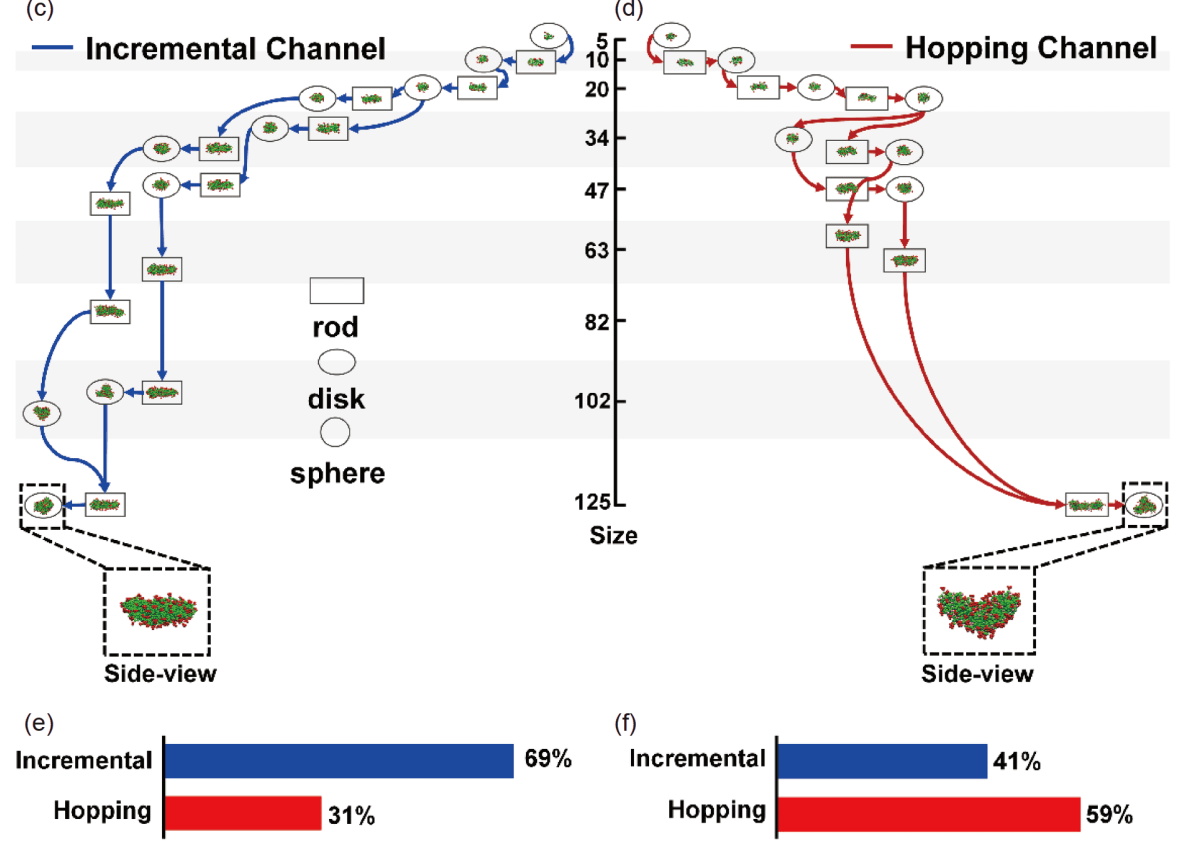

图 3 两亲性PYR和PYN以及它们的组装路径. (a, b) PYR和PYN分子结构; (c, d) PYR的逐渐增长路径通道(incremental channel)和PYN跳跃路径通道(hopping channel)中分别对应的两条最优路径以及中间体构型的快照(正视图). 其中，插图为最 终组装构型侧视图的放大图. (e, f) 两种自组装路径通道在PYR (e)及PYN (f)体系中所占的比例 ${ }^{[45]}$ (网络版彩图)

Figure 3 Amphiphiles PYR and PYN and their assembly pathways. (a, b) The structures of PYR and PYN. (c, d) The top two assembly pathways of the preferred path channel for PYR (incremental growth channel, blue) (c) and PYN (the hopping growth channel, red) (d) are given with the snapshots of all conformational states on top-view. An enlarged side-view of the final assembled structure is given for each system. (e, f) The populations of the incremental and hopping growth channels are given for PYR (e) and PYN (f), respectively [45] (color online). 
长型通道，30\%属于跳跃型增长通道(图3(e)). PYN体 系则呈现出相反趋势，即其中将近 $60 \%$ 的转移路径属 于跳跃型增长通道，40\%属于逐渐增长型通道(图3(f)). 进一步的分析表明，这些差异是由PYR和PYN的聚集 体在融合过程中的“黏附速率”(adhesion rate)不同所 致. PYN分子形成的聚集体之间的黏附速率约是同等 大小PYR分子聚集体黏附速率的两倍。两种分子不同 的亲疏水性决定了其黏附速率的差别. PYR分子中亲 水基团与疏水基团有明确的分界，从而PYR分子形成 的聚集体表面有更多的正电荷分布. 由于静电排斥作 用，这些正电荷增大了聚集体相互黏附过程中的自由 能势垒，从而导致了较小的黏附速率．而存在于PYN 分子疏水端的酰胺官能团，弱化了疏水相互作用的强 度，导致聚集体的堆积密度(packing density)变小，从 而导致聚集体的表面电荷密度较小，因此其具有较大 的黏附速率.

进一步发现, 相比逐渐增长型通道, 跳跃型通道中 最终形成的聚集体结构有更大的曲率，这是由于逐渐 增长型通道中聚集体是通过黏附质量较小的多体或单 体而逐步增大的，因此更容易调整聚集体的局部构型 而转变成各向均匀的形貌. 而跳跃型通道中则是两个 大小相似的聚集体碰撞黏附在一起，尺寸较大的两聚 集体融合过程中构型调整较难，更容易形成曲率大的 纳米结构.PYN体系中跳跃型通道占主导地位，从而 有更多曲率较大的聚集体形成，最终自组装成曲率较 大的囊泡结构，而PYR则自组装形成曲率较小的纳米 管状结构 ${ }^{[45]}$.

在该方案中，基于数百条微秒尺度的粗粒化分子 动力学模拟轨迹, 通过构建动力学网络模型, 将所有 轨迹中的构象分成若干个中间态; 然后利用转移路径 理论, 搜寻分子组装体形成过程的数千条动力学路径, 并通过路径整合方法得到跳跃增长型和逐步增长型两 类路径通道，以探究不同路径通道对分子组装体形成 过程的影响. 结果表明，两种结构相似的两亲性分子 因采取不同的路径通道导致最终分子组装体结构不 同. 以逐步增长型路径通道为主的分子最终自组装成 局部曲率较小的纳米管，以跳跃增长型路径通道为主 的分子最终形成曲率较大的囊泡; 两种分子对动力学 路径通道选择性的差异由分子自组装中间体间黏附速 率的差异决定, 而与自组装单体的构象无关. 此研究通 过比较两种分子组装体形成过程动力学路径通道的区
别, 揭示实验上两种分子自组装结构迥异的本质, 提出 动力学路径可控的两亲性分子组装体形成机制, 为实 现两亲性分子自组装结构的精准调控提供理论依 据 ${ }^{[4]}$

\section{3 嵌段共聚物自组装机制的研究}

\section{1 布朗动力学模拟两亲性嵌段共聚物自组装}

两亲性三维树枝状球形结构, 如由 $\mathrm{ABC}$ 三种成分 构筑的两亲性星型嵌段共聚物可在溶液中形成纳米 管、胶束、囊泡等，在超分子组装领域发挥着重要作 用 ${ }^{[60,61]}$. 吕中元教授等 ${ }^{[62]}$ 通过布朗动力学模拟研究了 两亲性星型多臂嵌段共聚物所处的溶剂环境、聚合物 链的构象与其组装结构之间的关系，以揭示溶剂条 件对分子组装结构的形成过程的影响. 图4(a)中显示 了星型多臂嵌段共聚物 $\left(\mathrm{A}_{x 1}\right)_{y}-\mathrm{C}-\left(\mathrm{B}_{x 2}\right)_{y}$ 的模型, $\mathrm{A}$ 和 $\mathrm{B}$ 代表两种聚合物链, $x 1$ 和 $x 2$ 代表链的长度, $y$ 代表链的 数目, $\mathrm{C}$ 代表中间连接点. 在此布朗动力学模拟中, 采用的是隐式溶剂模型. 成键参数采用谐振子势能, B-B、B-C和 $\mathrm{C}-\mathrm{C}$ 的非键相互作用采用Lennard-Jones $(\mathrm{LJ})$ 势能 $U_{\mathrm{LJ}}=4 \varepsilon\left[\left(\frac{\sigma}{r}\right)^{12}-\left(\frac{\sigma}{r}\right)^{6}\right]$, 为了描述 $\mathrm{A}$ 型珠子的不 同溶剂环境，对 $\mathrm{L} \mathrm{J}$ 势能函数进行修改 $U_{\mathrm{AA}}=4 \varepsilon\left[\left(\frac{\sigma}{r}\right)^{12}-\alpha\left(\frac{\sigma}{r}\right)^{6}\right]$, 其中 $\alpha$ 表示 $\mathrm{A}$ 型珠子亲/疏溶剂 的能力. $\alpha$ 取值范围为 $[0,1], \alpha$ 越小代表 $\mathrm{A}$ 的亲溶剂能力 越强, $\alpha=0$ 时此势能函数只有排斥项, 表示良溶剂; $\alpha=1$ 时, 势能函数为LJ势, 代表不良溶剂. 通过建立Flory指 数与 $\alpha$ 的关系, 结果表明 $\alpha \leq 0.4$ 代表良溶剂环境; $\alpha \geq 0.6$ 代表不良溶剂环境.

当 $\alpha=0$ ( $\mathrm{A}$ 的良溶剂), $y=5$ 时, 改变 $\mathrm{A} 、 \mathrm{~B}$ 链的长度, 发现不同链长的星型共聚物都可以形成球形胶束，且 稳定球形胶束的平均尺寸随链长的增加而减小. 临界 堆积参数(critical packing parameter, CPP) 常被用来表 征自组装结构 ${ }^{[63,64]} . \mathrm{CPP}=v /\left(a_{0} l_{\mathrm{c}}\right)$, 其中 $a_{0}$ 代表亲水链的 最优表面积, $v$ 和 $l_{\mathrm{c}}$ 分别代表疏水链的体积和临界长度. 一般情况下，球形胶束对应的 CPP值小于 $1 / 3$; 圆柱状 胶束的CPP为 $[1 / 3,1 / 2]$; 囊泡和双层膜对应的CPP值大 于 $1 / 2$. 如果将 $B$ 型链长固定为 10 , 溶剂都为 $B$ 的不良溶 剂, 则 $v$ 和 $l_{\mathrm{c}}$ 可近似看作常数, $a_{0}$ 成为决定 CPP取值的唯 
(a)
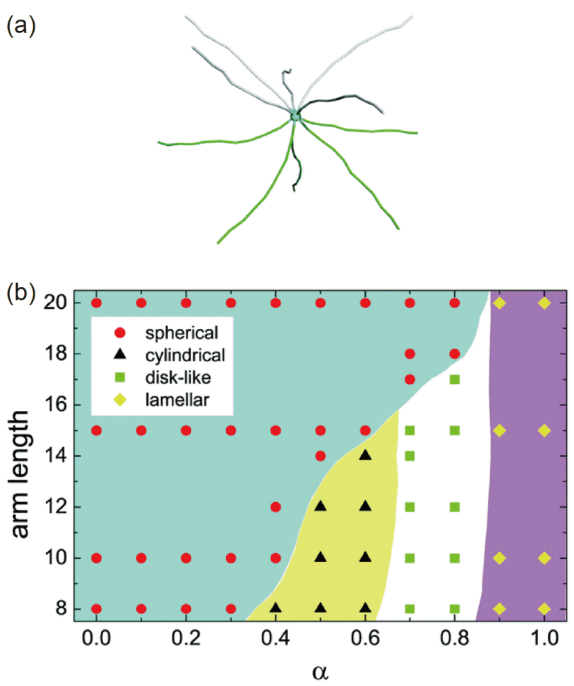

(c)

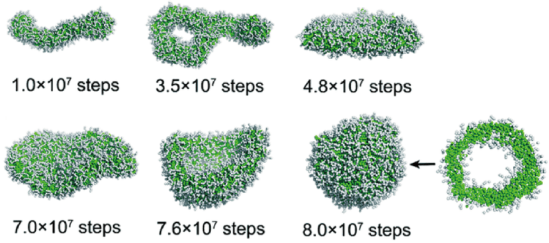

(d)

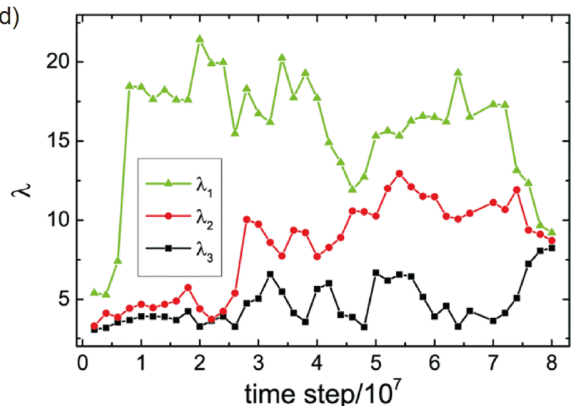

图 4 (a) 含有10个臂的星型嵌段共聚物结构. 其中, 浅灰色代表组成A, 浅绿色代表组成B, 连接A和B的中间珠子用青色表示. (b) 具有不同臂长和不同溶剂条件的多臂星状嵌段共聚物的形貌相图. (c) 体系 $\left(\mathrm{A}_{5}\right)_{5}-\mathrm{C}-\left(\mathrm{B}_{10}\right)_{5}$ 在 $\alpha=0.1$ 的条件下，自组装过程中 最大聚集体的典型构型的快照. 其中最后一个快照同时给出了其剖面图. (d) 体系 $\left(\mathrm{A}_{5}\right)_{5}-\mathrm{C}-\left(\mathrm{B}_{10}\right)_{5}$ 在 $\alpha=0.1$ 条件下, 自组装过程中 所形成的最大聚集体结构对应回旋张量半径的三个本征值 $\left(\lambda_{1}, \lambda_{2}\right.$ 和 $\left.\lambda_{3}\right)$ 随时间的变化 ${ }^{[62]}$ (网络版彩图)

Figure 4 (a) The structure of a miktoarm star-like block copolymer with 10 arms. Light gray arms are composed of component A, and green arms are composed of component B; the cyan bead is the center bead C. (b) The self-assembly morphology diagram of miktoarm star-like block copolymers with different arm lengths and in various solvent conditions for component A. (c) Typical structures found during key self-assembly events for the largest aggregate in the system of $\left(\mathrm{A}_{5}\right)_{5}-\mathrm{C}-\left(\mathrm{B}_{10}\right)_{5}$ when $\alpha=0.1$. The cross-sectional view of the vesicle is also shown for clarity. (d) The time evolution of $\lambda_{1}, \lambda_{2}$ and $\lambda_{3}$ of the largest aggregate in the system of $\left(\mathrm{A}_{5}\right)_{5}$-C-( $\left(\mathrm{B}_{10}\right)_{5}$ when $\alpha=0.1$ [62] (color online).

一变量. 当A成分的溶剂从良溶剂变成不良溶剂时, A 链会越来越塌缩, $a_{0}$ 减小, CPP增大. 图4(b)中展示了具 有不同臂长和不同溶剂条件的多臂星状嵌段共聚物的 形貌相图, 可以看出, 当 $\alpha$ 较小或A链较长时, 两亲性星 型嵌段共聚物自组装成球状胶束; 当链长较短且 $\alpha$ 增大 时, 圆柱状胶束开始形成; 当 $\alpha$ 继续增大时, 圆盘状胶束 开始形成; 而当 $\mathrm{A} 、 \mathrm{~B}$ 型链都疏溶剂时, 层状组装体 形成.

如果保持 $\mathrm{B}$ 链长度为 10 和疏溶剂的特征, 将亲溶 剂的 $A$ 链缩短为 5 可得到 $\left(\mathrm{A}_{5}\right)_{5}-\mathrm{C}-\left(\mathrm{B}_{10}\right)_{5}$, 模拟发现 $\mathrm{A} 、 \mathrm{~B}$ 两种成分链长不等且疏水端链更长时, $\left(\mathrm{A}_{5}\right)_{5}-\mathrm{C}-\left(\mathrm{B}_{10}\right)_{5}$ 嵌段共聚物在 $\alpha$ 为 $[0.1,0.7]$ 范围内都可以形成囊泡. 如 图4(c)所示，当 $\alpha=0.1$ 时星型嵌段共聚物先形成圆柱状 胶束, 然后变成圆柱状和圈状胶束, 再形成盘状胶束, 进而卷曲成碗状胶束, 最后形成封闭的球状囊泡. 图4 (d)中显示系统中最大尺寸聚集体对应回旋张量半径 的三个本征值 $\left(\lambda_{1}, \lambda_{2}\right.$ 和 $\left.\lambda_{3}\right)$ 随时间变化的情况 ${ }^{[65]}$, 可以看 出, 初试阶段 $\lambda_{1}>\lambda_{2} \approx \lambda_{3}$, 代表圆柱状胶束形成; 然后 $\lambda_{1} \approx \lambda_{2}>\lambda_{3}$, 代表盘状胶束形成; 最终三个本征值趋于相 等, 代表球形囊泡形成. 如果 $\alpha$ 的值在 $[0.2,0.7]$ 范围,
$\left(\mathrm{A}_{5}\right)_{5}-\mathrm{C}-\left(\mathrm{B}_{10}\right)_{5}$ 嵌段共聚物最终也可以形成球形囊泡, 与 $\alpha=0.1$ 不同的是, 在自组装的初始阶段形成的盘状胶 束而并非圆柱状胶束. 此工作为两亲性多臂星型嵌段 共聚物的自组装进行了系统的研究, 可以为实验上多 臂星型嵌段共聚物构筑基元的设计提供理论指导 ${ }^{[62]}$.

\section{2 构建动力学网络模型分析嵌段共聚物自组装 路径}

自组装过程中最终所形成的聚集体形貌不但取决 于体系的热力学性质, 还取决于体系的动力学性质. 结 合嵌段共聚物体系的动力学性质, 实验上已经通过多 阶层自组装过程获得稳定的自组装结构 ${ }^{[6]}$. 因此, 了 解嵌段共聚物的动力学信息, 更有助于设计自组装材 料和调控自组装材料的物理化学特性. 我们之前的研 究表明, 动力学网络模型可以揭示嵌段共聚物的主要 自组装路径, 发现控制自组装路径的关键参数, 从而 达到调控自组装路径的目的 ${ }^{[44]}$.

首先, 我们用动力学网络模型找到了星状嵌段共 聚物形成囊泡的主导组装路径(图5). 星状嵌段共聚物 如图 5(a)插图所示, 由 5 条长度为 10 的疏水链和 5 条长 
(a)

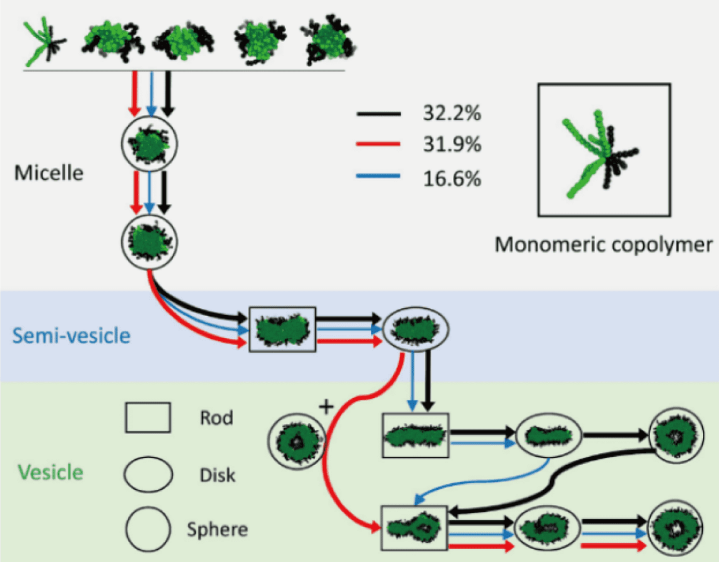

(b)

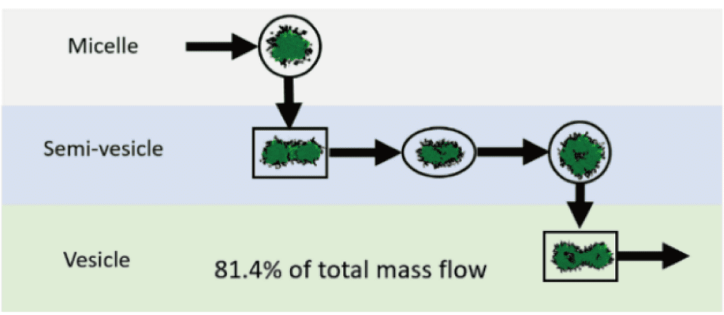

图 5 (a) 星状嵌段共聚物主导自组装路径. 其中包括三个 阶段：胶束阶段(浅灰色背景)、胶束融合阶段(浅蓝色背景) 以及囊泡阶段(浅绿色背景). (b) 调控后的中期主导路径. 大 部分盘状胶束转变成了半囊泡 ${ }^{[44}$ (网络版彩图)

Figure 5 (a) Three different stages of the aggregate growth of starlike copolymers are highlighted: micelle (grey), semi-vesicle (light blue), and vesicle (light green). (b) The dominant self-assembly pathway of the system with $\alpha=0.4$ [44] (color online).

度为 10 的亲水链构成. 其中 $\alpha=0.6$, 用来模拟不良溶剂. 我们选取了聚集体的大小以及非球形参数来划分不同 的态. 基于“质量流”矩阵，我们用修正的Dijkstra方 法 ${ }^{[48]}$ 找到了形成囊泡的主导路径. 结果发现，主导路 径可以分为三个阶段：初期胶束形成阶段、中期胶束 融合阶段以及未期囊泡形成阶段. 初期阶段，高分子 链聚集形成球状胶束, 胶束内部为嵌段共聚物的疏水 链, 外部为亲水链. 中期阶段, 不同的球形胶束碰撞融 合, 转变为盘状胶束. 末期阶段会形成较大的盘状胶 束, 它们进一步卷曲, 经过碗状结构的中间态, 最终闭 合形成囊泡结构. 我们注意到, 基于动力学网络模型找 到的末期阶段中囊泡形成过程，与之前研究结果一 致 ${ }^{[67 \sim 70]}$, 证明了我们方法的可靠性.

其次, 我们发现主导路径是由两种相互竞争的动 力学过程所控制：聚集体的碰撞过程以及聚集体形状 “棒 $\rightarrow$ 盘 $\rightarrow$ 球”的转变过程. 这两种动力学过程的快慢
可以分别用两个时间参数来表征：聚集体平均碰撞时 间 $T_{\mathrm{e}}$ 和聚集体转变时间 $T_{\mathrm{t}}$. 在初期胶束以及末期囊泡 阶段, $T_{\mathrm{t}}$ 远小于 $T_{\mathrm{e}}$, 表明聚集体在与另一个聚集体碰撞 融合之前有足够的时间转变成球形. 而在中期阶段, $T_{\mathrm{e}}$ 小于 $T_{\mathrm{t}}$, 因此, 中期阶段的聚集体在进入下一阶段之前 一直处于盘状, 并未转变成球形.

最后，基于上述机理，我们通过改变 $T_{\mathrm{e}}$ 和 $T_{\mathrm{t}}$ 的相对 大小, 调控了中期阶段的主导路径. 中期阶段中, 聚集 体由盘状转变成球状过程中的势垒导致 $T_{\mathrm{t}}$ 大于 $T_{\mathrm{e}}$. 中 期阶段中的球状结构为半囊泡(semivesicle), 实心结 构, 由内到外依次为亲水链、疏水链、亲水链所组成 的三层结构 ${ }^{[70 \sim 72]}$. 半囊泡是由盘状胶束表面的亲水链 扩散到内部疏水链所形成，与囊泡形成机制截然不同. 此过程中, 亲水链需要克服胶束内部较强的疏水作用 强度. 因此，中期阶段大部分聚集体并未转变成球状 结构. 我们通过改变 $\alpha$, 增大了亲-疏链的相对作用强 度, 进而加快了亲水链扩散速度, 减小了 $T_{\mathrm{t}}$. 最终, 如 图5(b)所示, 中期阶段大部分盘状胶束转变成了半 囊泡.

\section{4 补丁粒子自组装机制研究}

\section{1 粗粒化动力学模拟补丁粒子自组装}

目前，利用两亲性嵌段共聚物自组装已经能够获 得各种表面亲疏水性质可控的补丁粒子 ${ }^{[73]}$. 这些两亲 性粒子, 其尺寸可以跨越纳米到微米的范围, 能在更 大尺度上模拟原子和分子的两亲性行为, 为设计新型 自组装结构提供了新的方向. 通过合理设计补丁粒子 的软形变和表面各向异性特性, 可以得到非常丰富的 自组装结构, 如仿生螺旋结构、二维和三维的晶格结 构 $^{[74,75]}$. 为了实现在更大时间和空间尺度上有效模拟 软补丁粒子的聚集行为, 李等 ${ }^{[73,76]}$ 开发了软补丁粒子 模型(soft patchy particle models, SSPMs). 此模型利用 软各向异性吸引势函数来描述补丁粒子之间的相互 作用.

富勒烯 600 是由 60 个 $\mathrm{sp}^{2}$ 型碳原子形成的正二十面 体球, 其中包含 20 个六元环和 12 个五元环. C60含6-6和 6-5两种类型的键, 其中6-6键比6-5键短一些. 五元环和 六元环中的键角分别为 $120^{\circ}$ 和 $108^{\circ}, 6-6$ 键和五元环平 面的夹角为 $32^{\circ}$. 由于富勒烯型笼在结构和光电性能方 面的独特性，大量的科研工作者都在尝试合理设计构 
筑基元, 以通过自组装的方式获得多边形笼状构型.

为了研究超胶体笼状结构的设计原则与调控机 制, 李等 ${ }^{[77]}$ 用SPPMs设计了含三个补丁的补丁粒子(三 补丁粒子), 以模拟富勒烯型笼状结构的形成过程. 三 个补丁的相对位置由 $\Psi$ 和 $Q$ 所确定. 其中， $\Psi$ 是补丁粒 子中心指向第一和第二个补丁的两向量 $\mathbf{n}_{1}$ 和 $\mathbf{n}_{2}$ 的夹角, 本研究中, $\Psi=108^{\circ} . \Omega$ 是 $\mathbf{n}_{1}$ 和 $\mathbf{n}_{2}$ 所组成的平面与 $\mathbf{n}_{3}$ 形成 的夹角. 补丁大小由张角 $\theta$ 所确定, 其大小为补丁粒子 中心指向补丁面心及补丁边缘两向量夹角. 当 $\theta$ 为 0 时, 补丁大小为 0 , 当 $\theta$ 为 $90^{\circ}$ 时, 补丁大小为补丁粒子表面 积一半. 补丁粒子的相互作用可以通过与实验上的黏 合 $G$ 和弹性模量 $E$ 来设计. $G$ 可以决定补丁粒子间的相 互作用强度, $G$ 越大粒子间相互作用越强.

他们发现，通过调节补丁粒子大小、相互作用强 度及相对排列位置, 可以形成不同的结构, 如图6(a, b) 所示. 当补丁相对排列位置不变时 $\left(\Psi=108^{\circ}, \Omega=32^{\circ}\right)$, 补
丁较小 $\left(\theta\right.$ 位于 $\left[40^{\circ}, 50^{\circ}\right)$ 及相互作用较弱 $\left(G\right.$ 位于 $\left[2.5 k_{\mathrm{B}} T\right.$, $\left.3.5 k_{\mathrm{B}} T\right]$ ) 时，补丁粒子可形成类富勒烯笼状结构(图6 (c)). 当补丁较大时 $\left(\theta\right.$ 位于 $\left.\left[55^{\circ}, 50^{\circ}\right]\right)$, 类洋䓤笼状结构 (图6 (d) ) 可以在较大相互作用能量范围内 $(G$ 位于 $\left[2.5 k_{\mathrm{B}} T, 3.5 k_{\mathrm{B}} T\right]$ )形成. 其中, 粒子间相互作用更弱可以 形成无定型的自组装体，而当粒子间相互作用很强时， 形成类石墨烯曲面结构(图6(e)). 他们发现, 类富勒烯 笼状结构可以在较广范围的 $\Omega$ 下形成(图6(b)). 同时通 过调节 $\Omega$, 还可以形成其他结构(图6(f $\mathrm{h})$ ).

\section{2 构建动力学网络模型分析补丁粒子自组装 路径}

为了深入理解补丁粒子形成正十二面体类富勒烯 结构的机理, 我们构建了动力学网络模型找到了主导 自组装路径 ${ }^{[77]}$. 选取聚集体大小、五元环和六元环个 数这三个参数来划分态. 结果发现, 正十二面体类富
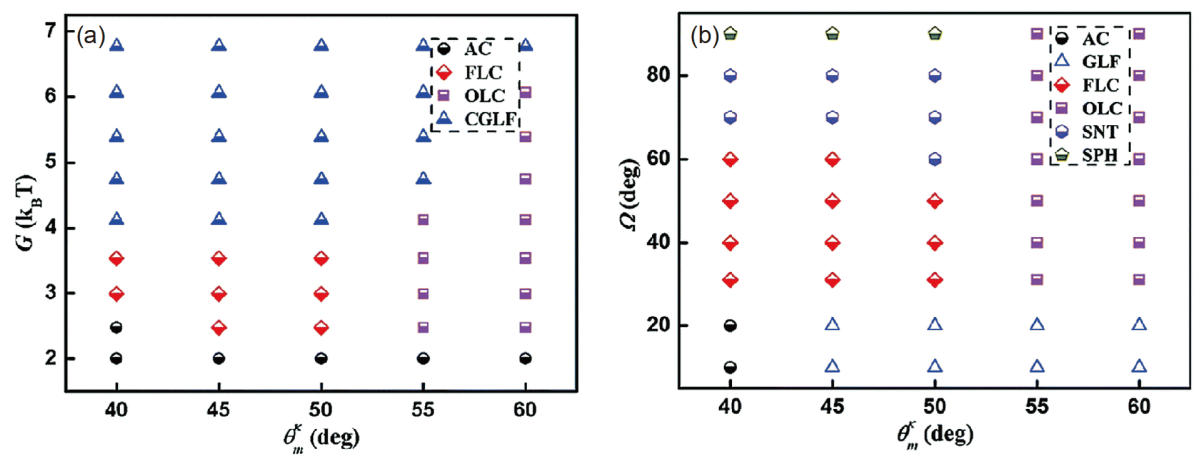

(c)

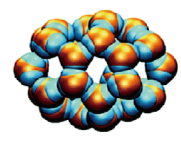

FLC

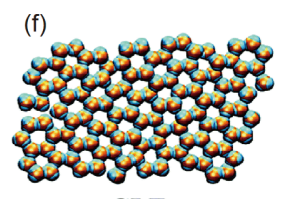

GLF (d)

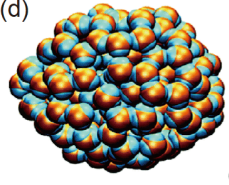

OLC

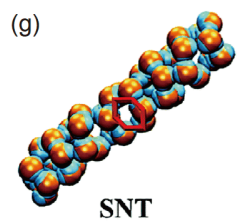

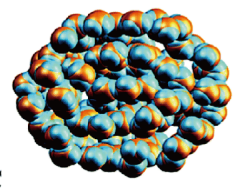

(e)

(h)
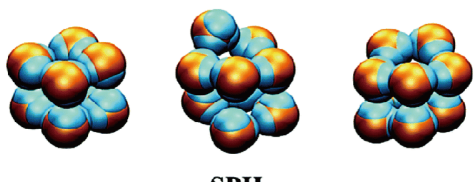

SPH

图 6 三补丁粒子所形成的不同形貌超胶束结构及相图. (a) $G-\theta$ 空间相图, 其中 $\Psi=108^{\circ}, \Omega=32^{\circ}, E=4.0 \mathrm{MPa}$. (b) $\Omega-\theta$ 空间相图, 其中 $\Psi=108^{\circ}, E=4.0 \mathrm{MPa}, G \approx 3.00 k_{\mathrm{B}} T$. (c f) 三补丁粒子所形成的不同形貌超胶束结构, 依次为: 无定型聚集体(ACs)、类富勒烯 笼状结构(FLC)、类洋葱笼状结构(OLCs)、类石墨烯曲面结构(CGLFs)、类石墨烯平面结构(GLFs)、细管状结构(SNTs)以及 小多面体结构(SPH) [77] (网络版彩图)

Figure 6 Supracolloidal fullerene-like cages self-assembled from soft three-patch particles by designing patch configuration, size, and interaction. (a) Phase diagram in the $G-\theta$ space, with $\Psi=108^{\circ}, \Omega=32^{\circ}, E=4.0 \mathrm{MPa}$. (b) Phase diagram in the $\Omega-\theta$ space at $\Psi=108^{\circ}, E=4.0 \mathrm{MPa}, G \approx 3.00 k_{\mathrm{B}} T$. (c) The different supracolloidal structures formed by three-patch particles, including amorphous clusters (ACs), fullerene-like cages (FLCs), onion-like cages (OLCs), curved graphene-like films (CGLFs), graphene-like films (GLFs), slender nanotube-like structures (SNTs), and small polyhedra (SPH) [77] (color online). 
勒烯形成包括两个阶段：聚集体增大和聚集体结构重 组(图7). 在聚集体增大阶段，多个补丁粒子聚集在一 起，达到了形成正十二面体所需要的补丁粒子数目. 此阶段中，聚集体有各种无定形的构象，并有少量五 元环或六元环. 在结构重组阶段, 聚集体停止增长, 五 元环数量逐渐增多至 12 , 六元环数量则相应减少到 0 , 最终形成正十二面体类富勒烯笼状结构。此处，通过 动力学网络模型得到的类富勒烯笼状结构形成机制, 验证了之前通过观察动力学轨迹所得到的结论 ${ }^{[77]}$. 值 得一提的是, 此笼状结构的形成与囊泡形成十分相似, 都是由片状结构卷曲, 通过中间碗状结构, 最终闭合形 成囊泡或者笼状结构。补丁粒子所形成的片状和碗状 结构分别如图7插图和其中标号9、11所示. 我们发现, 在正十二面体笼状结构过程中，补丁粒子还可以形成 大于 20 的片状结构进而闭合形成更大的笼状结构，这 些笼状结构的形成严重限制了正十二面体结构的产 率. 因此, 我们设计了一种可以尽早触发和加快结构重

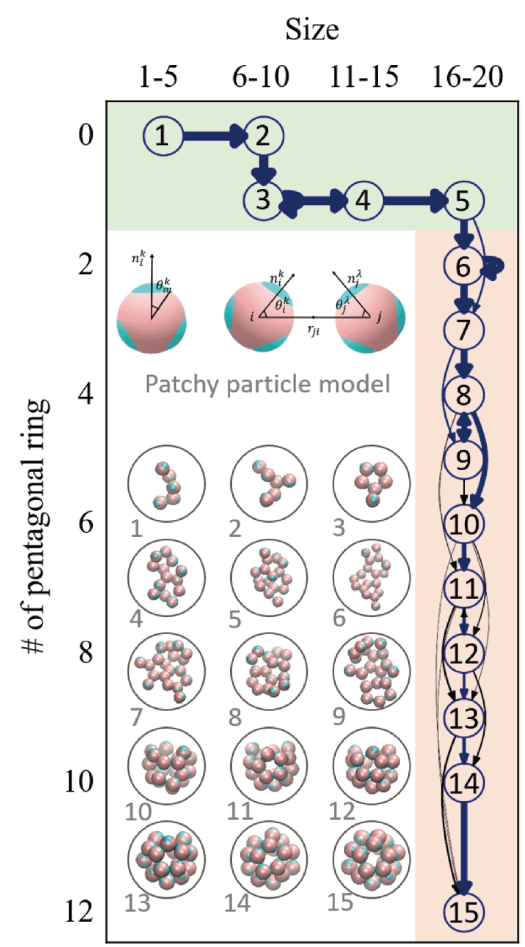

图 7 正十二面体类富勒烯的形成路径. 包括两个阶段: 聚 集体增大(浅绿色背景)和聚集体结构重组(粉红背景)。插图 为补丁粒子模型以及每个态的代表构象 ${ }^{[78]}$ (网络版彩图)

Figure 7 The self-assembled process of the dodecahedral cage formation, including aggregate size increasing stage (light green) and the structural rearrangement stage (light red). Inset shows the snapshot of the representative conformations of each state [78] (color online).
组过程的补丁粒子，从而避免了聚集体在形成正十二 面体之前继续增大，进一步提升了正十二面体笼状结 构的产率. 通过结合动力学模拟与动力学网络模型分 析，我们提出了一种新的基于动力学分析的超胶体结 构设计理念 ${ }^{[78]}$. 此项研究为实验上获取类富勒烯笼状 结构提供了新的思路, 同时为深入理解富勒烯、病毒 衣壳等笼状结构形成机制提供了新的视角.

\section{5 总结与展望}

本文介绍了我们近期在两亲性体系自组装机制的 多尺度模拟和动力学网络模型分析方面的相关研究进 展，展现了多尺度动力学模拟在模拟自组装结构形成 过程、探索自组装内在机制方面的重要性，同时也证 明了动力学网络模型在研究自组装体系动力学方面的 巨大潜力.

通过多尺度分子动力学模拟，可以针对自组装过 程的不同层次, 适应性地模拟自组装结构的形成过程, 将微观和介观尺度分子组装过程可视化, 弥补实验上 组装过程表征的空白, 并且粗粒化动力学模拟所得结 构也能够向全原子映射, 这样可以直接建立原子尺度 纳米组装体与构筑基元的结构细节，对实验上构筑基 元的设计和组装结构的调控有很大的启发.

通过动力学网络模型分析, 我们可以得到自组装 体系中具有统计意义的动力学信息, 如自组装路径. 需要强调的是, 我们此处发展的动力学网络模型分析 方法不仅适用于文中所提到的两亲性有机小分子、嵌 段共聚物及补丁粒子等体系, 还适用于其他自组装体 系, 如寡肽的自组装及病毒衣壳的自组装.

同时，值得注意的是，目前基于聚集体的物理性 质，如大小、形貌所构建的动力学网络模型还有一定 局限性. 构建“质量流”矩阵时，聚集体 $\mathrm{A}$ 和B碰撞融合 成 $\mathrm{C}$ 这一事件被分隔成的 $\mathrm{A}$ 到 $\mathrm{C}$ 和 $\mathrm{B}$ 到 $\mathrm{C}$ 两个独立事件. 因此根据“质量流”矩阵寻找聚集路径时，忽略了路径 之间的关联性. 这种关联性是由基于单个聚集体结构 性质划分构象空间所引起的。一种可行的考虑路径间 关联性的方案是根据体系的整体结构性质来划分构象 空间. 体系某一时刻整体结构可用此时刻体系内所有 聚集体大小及形状的分布来表征. 不同时刻下体系的 结构差别可以用此分布的差异来表征，进而根据分布 差异来划分小态, 更合理地构建转移概率矩阵和动力 
学网络模型. 此方案的可行性及可靠性还有待进一步 研究.

在我们所构建的动力学网络模型中, 用“质量流” 来近似表示自组装体系不同态之间的动力学信息. 由 于自组装体系的“不可逆性”, 不能直接根据“质量流” 矩阵来获取不同态的稳定构象数量 (population of stable states), 因此不能求得态的相对自由能大小. 为 了准确获取不同态的稳定构象数量及相对自由能大 小, Frenkel等 ${ }^{[15,47]}$ 用Wang-Landau方法 ${ }^{[79,80]}$ 获取了
DNA块状模型自组装体系中各个态的态密度, 从而得 到了态的自由能大小. 此方法也适用于其他自组装体 系. 为了准确计算模拟中稀有事件(rare event)发生的 概率, Noé等 ${ }^{[81,82]}$ 和Hummer等 ${ }^{[83]}$ 发展了一系列Tram方 法来分析增强采样数据, 获取体系不同态之间的转移 概率, 进而可以得到不同态之间的动力学信息及稳定 构象数量. Tram方法有望应用在自组装体系中, 来获 得自组装结构的解组装过程发生概率, 进一步帮助我 们构建更加精准的动力学网络模型.

\section{参考文献}

1 Wang Y, Xu H, Zhang X. Adv Mater, 2009, 21: 2849-2864

2 Zhang X, Wang C. Chem Soc Rev, 2011, 40: 94-101

3 Yang L, Tan X, Wang Z, Zhang X. Chem Rev, 2015, 115: 7196-7239

4 Yu G, Jie K, Huang F. Chem Rev, 2015, 115: 7240-7303

5 Wang J, Liu K, Xing R, Yan X. Chem Soc Rev, 2016, 45: 5589-5604

6 Liu H, Zhang H, Hu Z, Qian H, Lu Z. Sci Sin Chim, 2019, 49: 299-310 (in Chinese) [刘鸿, 张厚玉, 胡中汉, 钱虎军, 吕中元. 中国科学: 化学, 2019, 49: 299-310]

7 Gubbins KE, Moore JD. Ind Eng Chem Res, 2010, 49: 3026-3046

8 Maginn EJ, Elliott JR. Ind Eng Chem Res, 2010, 49: 3059-3078

9 Müller M, Katsov K, Schick M. Phys Rep, 2006, 434: 113-176

10 Frenkel D, Smit B. Understanding Molecular Simulation: From Algorithms to Applications. Singapore: Elsevier Pte Ltd., 2001

11 Bryngelson JD, Onuchic JN, Socci ND, Wolynes PG. Proteins, 1995, 21: 167-195

12 Dill KA, Chan HS. Nat Struct Mol Biol, 1997, 4: 10-19

13 Dill KA, MacCallum JL. Science, 2012, 338: 1042-1046

Jacobs WM, Reinhardt A, Frenkel D. Proc Natl Acad Sci USA, 2015, 112: 6313-6318

Noé F, Fischer S. Curr Opin Struct Biol, 2008, 18: 154-162

Wang W, Cao S, Zhu L, Huang X. WIREs Comput Mol Sci, 2018, 8

Bowman GR, Huang X, Pande VS. Methods, 2009, 49: 197-201

Zhu L, Sheong FK, Zeng X, Huang X. Phys Chem Chem Phys, 2016, 18: 30228-30235

Prinz JH, Wu H, Sarich M, Keller B, Senne M, Held M, Chodera JD, Schütte C, Noé F. J Chem Phys, 2011, 134: 174105

Husic BE, Pande VS. J Am Chem Soc, 2018, 140: 2386-2396

Noé F, Schütte C, Vanden-Eijnden E, Reich L, Weikl TR. Proc Natl Acad Sci USA, 2009, 106: 19011-19016

Voelz VA, Bowman GR, Beauchamp K, Pande VS. J Am Chem Soc, 2010, 132: 1526-1528

Zhou S, Wang Q, Wang Y, Yao X, Han W, Liu H. Phys Chem Chem Phys, 2017, 19: 11249-11259

Da LT, Pardo Avila F, Wang D, Huang X. PLoS Comput Biol, 2013, 9: e1003020

Da LT, Pardo-Avila F, Xu L, Silva DA, Zhang L, Gao X, Wang D, Huang X. Nat Commun, 2016, 7: 11244

Silva DA, Weiss DR, Pardo Avila F, Da LT, Levitt M, Wang D, Huang X. Proc Natl Acad Sci USA, 2014, 111: 7665-7670

Zhang L, Pardo-Avila F, Unarta IC, Cheung PPH, Wang G, Wang D, Huang X. Acc Chem Res, 2016, 49: 687-694

Silva DA, Bowman GR, Sosa-Peinado A, Huang X. PLoS Comput Biol, 2011, 7: e1002054

Gu S, Silva DA, Meng L, Yue A, Huang X. PLoS Comput Biol, 2014, 10: e1003767

Buch I, Giorgino T, de Fabritiis G. Proc Natl Acad Sci USA, 2011, 108: 10184-10189 
Held M, Metzner P, Prinz JH, Noé F. Biophys J, 2011, 100: 701-710

Da LT, Lin M. Phys Chem Chem Phys, 2019, 21: 26003-26016

Kuzmanic A, Bowman GR, Juarez-Jimenez J, Michel J, Gervasio FL. Acc Chem Res, 2020, 53: 654-661

Qiao Q, Bowman GR, Huang X. J Am Chem Soc, 2013, 135: 16092-16101

Qiao Q, Qi R, Wei G, Huang X. Phys Chem Chem Phys, 2016, 18: 29892-29904

Yang YI, Gao YQ. J Phys Chem B, 2014, 119: 662-670

Cao Y, Jiang X, Han W. J Chem Theor Comput, 2017, 13: 5731-5744

Weinan E, Vanden-Eijnden E. Annu Rev Phys Chem, 2010, 61: 391-420

Metzner P, Schütte C, Vanden-Eijnden E. Multiscale Model Simul, 2009, 7: 1192-1219

Zeng X, Zhu L, Zheng X, Cecchini M, Huang X. Phys Chem Chem Phys, 2018, 20: 6767-6776

Peng J, Wang W, Yu Y, Gu H, Huang X. Chin J Chem Phys, 2018, 31: 404-420

Sengupta U, Carballo-Pacheco M, Strodel B. J Chem Phys, 2019, 150: 115101

Zeng X, Li B, Qiao Q, Zhu L, Lu ZY, Huang X. Phys Chem Chem Phys, 2016, 18: 23494-23499

Zheng X, Zhu L, Zeng X, Meng L, Zhang L, Wang D, Huang X. J Phys Chem Lett, 2017, 8: 1798-1803

Perkett MR, Hagan MF. J Chem Phys, 2014, 140: 214101

Jacobs WM, Reinhardt A, Frenkel D. J Chem Phys, 2015, 142: 021101

Gupta A, Zangrilli M, Sundararaj AI, Huang AI, Dinda PA, Lowekamp BB. Free network measurement for adaptive virtualized distributed computing. In: Proceedings 20th IEEE International Parallel \& Distributed Processing Symposium. Rhodes Island: IEEE, 2006

Meng L, Sheong FK, Zeng X, Zhu L, Huang X. J Chem Phys, 2017, 147: 044112

Wang Y, Ma N, Wang Z, Zhang X. Angew Chem Int Ed, 2007, 46: 2823-2826

Tessier MB, Demarco ML, Yongye AB, Woods RJ. Mol Simul, 2008, 34: 349-364

Wang J, Wolf RM, Caldwell JW, Kollman PA, Case DA. J Comput Chem, 2004, 25: 1157-1174

Zheng X, Wang D, Shuai Z, Zhang X. J Phys Chem B, 2012, 116: 823-832

Kästner J. WIREs Comput Mol Sci, 2011, 1: 932-942

Zheng X, Wang D, Shuai Z. Nanoscale, 2013, 5: 3681-3689

Marrink SJ, Risselada HJ, Yefimov S, Tieleman DP, de Vries AH. J Phys Chem B, 2007, 111: 7812-7824

Arai N, Yasuoka K, Zeng XC. J Am Chem Soc, 2008, 130: 7916-7920

Wang C, Yin S, Chen S, Xu H, Wang Z, Zhang X. Angew Chem, 2008, 120: 9189-9192

Wang C, Chen Q, Xu H, Wang Z, Zhang X. Adv Mater, 2010, 22: 2553-2555

Yan D, Zhou Y, Hou J. Science, 2004, 303: 65-67

Zhou Y, Yan D. Angew Chem Int Ed, 2004, 43: 4896-4899

Li B, Zhao L, Qian HJ, Lu ZY. Soft Matter, 2014, 10: 2245-2252

Lin CM, Wu DT, Tsao HK, Sheng YJ. Soft Matter, 2012, 8: 6139-6150

Yin H, Kang SW, Bae YH. Macromolecules, 2009, 42: 7456-7464

Lin CM, Chen YZ, Sheng YJ, Tsao HK. Reactive Funct Polyms, 2009, 69: 539-545

Gröschel AH, Schacher FH, Schmalz H, Borisov OV, Zhulina EB, Walther A, Müller AHE. Nat Commun, 2012, 3: 710

Noguchi H, Takasu M. Phys Rev E, 2001, 64: 041913

Yamamoto S, Maruyama Y, Hyodo S. J Chem Phys, 2002, 116: 5842-5849

Han Y, Yu H, Du H, Jiang W. J Am Chem Soc, 2010, 132: 1144-1150

He X, Schmid F. Phys Rev Lett, 2008, 100: 137802

He X, Schmid F. Macromolecules, 2006, 39: 2654-2662

Xiao M, Xia G, Wang R, Xie D. Soft Matter, 2012, 8: 7865-7874

Li ZW, Zhu YL, Lu ZY, Sun ZY. Soft Matter, 2016, 12: 741-749

Du J, O’Reilly RK. Chem Soc Rev, 2011, 40: 2402

Glotzer SC. Science, 2004, 306: 419-420

Li ZW, Sun ZY, Lu ZY. Simulation models of soft janus and patchy particles. In: Yan LT, Ed. Self-Assembling Systems: Theory and Simulation. 
Chapter 5. Weinheim: JohnWiley \& Sons, 2017. 109-133

\title{
Multiscale simulation and kinetic network model analysis of the self- assembly of amphiphilic systems
}

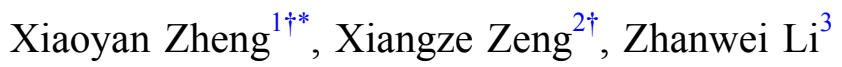 \\ ${ }^{1}$ Beijing Key Laboratory of Photoelectronic/Electrophotonic Conversion Materials, Key Laboratory of Cluster Science of Ministry of Education, \\ School of Chemistry and Chemical Engineering, Beijing Institute of Technology, 100081 Beijing, China; \\ ${ }^{2}$ Department of Biomedical Engineering, Washington University in St. Louis, St. Louis, MO, 63130, USA; \\ ${ }^{3}$ State Key Laboratory of Polymer Physics and Chemistry, Changchun Institute of Applied Chemistry, Chinese Academy of Sciences, Changchun \\ 130022, China \\ $\dagger$ These authors contributed equally to this work. \\ *Corresponding author (email: xiaoyanzheng@bit.edu.cn)
}

\begin{abstract}
Amphiphilic building blocks play an important role in fabricating nanomaterials. To achieve the precise control of self-assembled nanostructures, it is important to understand the self-assembly process at both microscopic and mesoscopic scale. Multiscale simulations could simulate the self-assembly process in both microscopic and mesoscopic scale, and get the metastable and equilibrium assembled structures and the corresponding trajectories. Based on these trajectories, we can further build the kinetic network model and obtain the kinetic transition pathway of the selfassembly process. This approach provides the theoretical principles for designing novel self-assembly building blocks and achieving novel self-assembled structures. In this article, we review our recent progress on the study of mechanisms of self-assembly of amphiphilic building blocks, such as small organic molecules, block copolymers and patchy particles by combining multiscale simulation and kinetic network model analysis.
\end{abstract}

doi: $10.1360 /$ SSC-2020-0082 